\title{
La formación de la cultura matemática y sus dimensiones
}

\section{Yupayniyuu yaćhaynin yaćhachina pampanninkunawan}

\section{Kara okantagani ora ogokoitane matematika aike ora okanagekera}

Recepción: 19 mayo 2020 Corregido: 16 junio 2020 Aprobación: 04 noviembre 2020

Eneida Antonia Terry Leonard

Nacionalidad: Cubana / Universidad Carlos Rafael Rodríguez de Cienfuegos Correo: eaterry@ucf.edu.cu / ORCID: https://orcid.org/oooo-0001-8853-5912

Longino Ramón Muñoz del Sol Nacionalidad: Cubana / Universidad Carlos Rafael Rodríguez de Cienfuegos Correo: Irsol@ucf.edu.cu / ORCID: https://orcid.org/oooo-ooo2-3159-7124

Lourdes María Martínez Casanova

Nacionalidad: Cubana / Universidad Carlos Rafael Rodríguez de Cienfuegos Correo: Immartinez@ucf.edu.cu / ORCID: https://orcid.org/oooo-0002-1789-3891

\section{Resumen}

Es misión del profesor contribuir con la formación cultural e integral del estudiante. La formación de la cultura matemática constituye una necesidad en el proceso de enseñanza aprendizaje de la Licenciatura en Educación, Matemática, el futuro profesor debe ser el guía fundamental en dicho proceso y estar preparado para ejercer su profesión, para resolver problemas y comprender con cientificidad los fenómenos de la vida. Se ofrece una revisión teórica sobre la cultura matemática, con el objetivo de encontrar los aspectos que la caracterizan y establecer una vía para su formación, se recurre para ello al criterio de los expertos.

\section{Palabras clave:}

Formación, cultura

matemática, dimensiones.

\section{Lisichiku limaykuna:}

yaćhachina, yupayniyu

yaćhay, pampamkuna

Nibarintsipage katingatsaro: Okantagani ogotagantsipage, kengagantsi, Ogokoitane, 


\section{The Formation of the Mathematical Culture and Dimensions}

\begin{abstract}
It is the teacher's mission to contribute to the cultural and integral formation of the student. The formation of the mathematical culture constitutes a need in the process of apprenticeship teaching of the licentiate in mathematical education, the future teacher must be the fundamental guide in this process and is prepared to practice your profession, to resolve problems and understand with scientificity the phenomenona of the life. It offers a theoretical revision about the mathematical culture, with the objective to find the aspects that the characterize and establish a path for their formation, by appealing for it to the criterion of the experts.
\end{abstract}

\section{Keywords}

Formation, mathematical

culture, dimensions.

\section{A formação da cultura matemática e suas dimensões}

\section{Resumo}

É missão do professor contribuir para a formação cultural e integral do estudante. A formação da cultura matemática constitui uma necessidade no processo ensino-aprendizagem da licenciatura em Educação Matemática. O futuro professor deve ser o guia fundamental neste processo e estar preparado para exercer sua profissão, resolver problemas e compreender cientificamente os fenômenos da vida. É oferecida uma revisão teórica da cultura matemática, com o objetivo de encontrar os aspectos que a caracterizam e estabelecer uma via para a sua formação, recorrendo para isso aos critérios de especialistas.

\author{
Palavras-chave: \\ Formação, cultura \\ matemática, dimensões.
}

\section{Datos de los autores}

Eneida Antonia Terry Leonard es docente e investigadora en educación, matemática. Profesor asistente. Máster en Matemática Aplicada por la Universidad Carlos Rafael Rodríguez de Cienfuegos.

Longino Ramón Muñoz del Sol es docente y consultante en educación, matemática. Profesor titular. Doctor en Ciencias Pedagógicas por la Universidad Carlos Rafael Rodríguez de Cienfuegos.

Lourdes María Martínez Casanova es docente y consultante en educación, matemática. Profesor titular. Máster en Matemática Aplicada por la Universidad Carlos Rafael Rodríguez de Cienfuegos. Doctor en Ciencias Pedagógicas por la Universidad Carlos Rafael Rodríguez de Cienfuegos. 


\section{Introducción}

El análisis de las fuentes bibliográficas constata la existencia de una gran preocupación por el mejoramiento del estudio de la matemática y la formación de su cultura en los profesionales, en especial de la Licenciatura en Educación Matemática.

El esmero por el mejoramiento de la enseñanza de la matemática se torna evidente en China, donde se implementan técnicas con este objetivo, como en el programa 'Inspire Maths', utilizado ampliamente en Singapur y Shanghai, extendido a Inglaterra, Gran Bretaña y con ese mismo objetivo laboran en Chile, según los autores Soto (2015) y Rocío (2016).

El desarrollo científico, la utilización de libros de texto de alta calidad, el acceso a las tecnologías de comunicación e información y la enseñanza de una Didáctica de la Matemática para la construcción del conocimiento por los alumnos, benefician el proceso de enseñanza aprendizaje de la matemática y el desarrollo profesional de los docentes, sin embargo, no se enfatiza en la formación de la cultura matemática de los mismos en el nivel superior.

La cultura matemática es un tema tratado en la actualidad del ámbito internacional. De Guzmán (1993), Bishop (1999), Molina (2003), Mingüer y Lezama (2007) y Cantoral (2013) tratan aspectos teóricos de la enseñanza de la matemática que contribuyen a la formación de una cultura de esta ciencia, refiriéndose a las dificultades que se presentan en la enseñanza, la importancia de la historia de la matemática, del componente lúdico y de la teoría socio epistemológica. Precisan así los aspectos que se deben tratar para contribuir a la cultura matemática, pero no destacan su importancia en la preparación del profesor, ni proponen vías para lograrla.

En la revisión de los documentos normativos se constata que el modelo del profesional de la Licenciatura en Educación Matemática, plan E de las universidades cubanas tiene entre sus objetivos la formación de la cultura matemática solamente en las disciplinas Álgebra y Análisis Matemático de las específicas del currículo, sin describirse en sus programas cómo lograrlo.

Al aplicar métodos empíricos como el análisis de 10 textos básicos y de consulta de la carrera, la observación al proceso de enseñanza aprendizaje y entrevistas encubiertas a profesores y estudiantes de Matemática de la Universidad 'Carlos Rafael Rodríguez' de Cienfuegos y la Universidad Central 'Marta Abreu' de Las Villas, permiten apreciar que existen insuficiencias en el proceso y su concreción en la formación de la cultura matemática, manifestadas en los términos siguientes:

- No existe una percepción pedagógica de la necesidad de instrumentar la cultura matemática en el currículo escolar como parte importante de la formación profesional.

- Dada la preocupación por la enseñanza de la Matemática en el mundo no se concreta el accionar para la formación de la cultura matemática en los futuros profesionales de la educación de esta ciencia.

- El resultado de las clases observadas evidencia limitaciones en la aplicación o extrapolación de procedimientos de las disciplinas específicas a la solución de problemas de la vida práctica y su vinculación con otras ciencias y ramas del saber académico y cotidiano.

- Las orientaciones en el programa de las disciplinas específicas, sobre cómo formar la cultura matemática en los estudiantes de la Licenciatura en Educación, Matemática, son escasas. 
- Los docentes entrevistados no refieren con precisión y fundamentos sólidos las acciones dirigidas a la adquisición de una cultura matemática en sus estudiantes.

- No se aprovechan las potencialidades de los contenidos de las disciplinas específicas de la carrera para sistematizar los temas culturales, sino que se presentan con un carácter anecdótico y sin las premisas didácticas para llevarlo a cabo.

Al considerar la cultura matemática como uno de los aspectos más importantes a alcanzar y en especial en la carrera Licenciatura en Educación Matemática, se tiene como objetivo precisar los aspectos que caracterizan su formación en dicha carrera, para lo cual se aplicó una encuesta a expertos

\section{La cultura matemática y su formación}

Núñez (1999) afirma que la cultura es un mecanismo de regulación social, y en su interior, la ciencia se comporta como una subcultura sostenida por la actividad comunal de grupos practicantes. En el proceso de enseñanza aprendizaje se contribuye al conocimiento de diferentes ciencias que a la vez pueden considerarse como subculturas.

Diferentes autores han investigado y formulado propuestas acerca de la cultura energética Pupo (2006), la cultura económica Carralero, Tamayo y Alonzo (2013), Palanqué, Toledo, Torres, Cables y Almaguer (2015), Bello, Machado y López (2015), la cultura política Raya (2002) y Valdivia (2012), la cultura científica Salazar (2004). De manera general se refieren a un conjunto de conocimientos, actitudes, valores y experiencias con relación al campo que tratan.

Otros hacen referencia a la cultura matemática, tal es el caso de Molina (2003), el proyecto PISA/OECD (2004), Mingüer y Lezama (2007) y Cantoral (2013) quienes destacan en sus definiciones la capacidad o aptitud de un individuo, el conocimiento matemático, el producto de un conjunto de construcciones sucesivas de prácticas sociales vinculadas a la actividad matemática, o en última instancia, "lo que todavía recordamos cuando ya hemos olvidado todo lo que nos obligaron a aprender" (Sánchez, 2015, p. 3), no obstante, no se aprecian los aspectos esenciales que pueden constituir fundamentos útiles para formar la cultura matemática teniendo en cuenta su complejidad y carácter multidimensional.

Molina (2003), expone componentes de la cultura matemática, y son centrados en los conocimientos matemáticos del profesor, las habilidades que él demuestra en el aula, principalmente al utilizar la solución de problemas, y las actitudes positivas que tiene con los alumnos, tales como ser amable y respetuoso; sin embargo estos componentes no son suficientes para formar la cultura matemática de los estudiantes, sino se tiene en cuenta qué aspectos debe conocer el alumno y por ende el profesor, que los llevan a conocer la matemática en su plenitud.

Esta autora promueve la reflexión sobre el término cultura matemática cuando plantea “... de la variedad de características que lo describirán dependerá el punto de vista que se estudie" (Molina, 2003, p. 185).

En este sentido se analiza que a la formación de la cultura matemática se puede contribuir cuando se tiene en cuenta diferentes aspestos. El aspecto histórico de la matemática es tratado por varios autores como León (1999) y Arteaga (2017), quienes muestan la importancia que tiene la historia de la matemática en la enseñanza de la misma, para un mayor conocimiento de sus con- 
ceptos, teorías y leyes, así como para motivar al estudiante en el mundo del razonamiento lógico.

Los estudios realizado por otros autores Ilevan al camino de transformar la realidad en diferentes contextos aplicando la matemática, como es el caso de Camarena (2009) y Tones (2015) quienes con sus trabajos muestran la articulación de la matemática con diferentes carreras técnicas, de ingenierías, informática y otras, la elaboración de obras arquitectónicas.

Sin embargo, autores como García, Buitrago y Cañon (2015) y Fernández (2017), dejan ver otro aspecto que caracteriza a la cultura matemática al relacionarla con los juegos, acertijos, la música, Ia literatura, recursos didáctico y motivacional, con la utilización de medios informáticos (software educativos, asistentes matemáticos), transformando así el significado de la formación académica de una manera radical lo que ha incidido, de forma directa y positiva, en la evolución de la enseñanza tradicional.

Se constata la existencia de Investigaciones, Lezama y Mariscal (1995), Blanco, Fernández y Oliveras (2017) que tratan solo aspectos cognitivos como el dominio del contenido, las alternativas de solución, problemas profesionales sustentados en la apropiación del conocimiento, habilidades, valores, la logicidad del pensamiento, las prácticas matemáticas de grupos sociales, la epistemología de esta ciencia, pero para los futuros profesionales de la Educación Matemática deben considerarse, además, aspectos formativos que se expresan en el buen uso de los métodos, procedimientos, algoritmos para la enseñanza y los modos de actuación y valores profesionales del docente.

Autores como Rodríguez, Correa y Hernández (2014) y Moreno (2017) utilizan el término dimensión para tratar aspectos que caractericen un objeto a investigar, así lo reafirma Álvarez (1999) al plantear, que la dimensión es la proyección de un objeto o atributo en una cierta dirección. Según Horruitiner (2008) la dimensión expresa la perspectiva bajo la cual se analiza determinado proceso en circunstancias específicas. Expresado de esta manera, el concepto posibilita la caracterización parcial, específica, de cada uno de los procesos, atendiendo al rasgo (o rasgos) que en ese orden de ideas es fundamental para el sujeto que lo analiza.

Por lo que, al considerar que la cultura matemática muestra las diferentes direcciones en que puede ser vista la actividad matemática como cultura en la sociedad, se considera estas direcciones o aspectos como dimensiones que caracterizan la cultura matemática. Es decir que un mismo proceso se puede estudiar desde diferentes dimensiones, de modo que en cada una de ellas su comportamiento externo o función evidencie el aspecto que se desea connotar.

Se consideran los estudios afrontados por Coriat y Flores (1998) quienes refieren la cultura escolar de los estudiantes en formación inicial de la Licenciatura de Matemática de Granada y la definen como aquella que influye en la actitud de los profesores cuando afrontan los procesos institucionales de formación.

Estos autores proponen estudiar las culturas escolares desde diferentes perspectivas y atribuirles dimensiones estructurales:

- Dimensión Laboral- profesional

- Dimensión Educativa

- Dimensión Social

Estas dimensiones se encaminan a afrontar vivencias, dificultades y problemas debido a su situación profesional, a las decisiones operativas sobre las relaciones entre profesor y alumnos, y 
a las relaciones entre el entorno y las expectativas educativas del Claustro. Muy lejos esto, de la aplicación que tuvo y tiene en la actualidad la matemática, del conocimiento individual y personal sobre la génesis y surgimiento de la asignatura que imparte y de la formación que debe tener como docente este futuro profesional de la enseñanza de la matemática; es decir, de su cultura matemática como parte de su formación integral.

Por lo que, al considerarse la cultura matemática como una de las aristas esenciales del desarroIlo de la cultura general integral de los estudiantes docentes en formación, es una necesidad insoslayable analizar su proceso de formación a partir de estos aspectos que la autora sugiere llamarle dimensiones que caracteriza la cultura matemática.

De manera general, se observa cómo la cultura matemática vista desde los aspectos o dimensiones presentadas por los autores consultados tiene un espectro amplio que puede ser tratado en el proceso de enseñanza aprendizaje de las disciplinas específicas de la Licenciatura en Educación Matemática, propiciando así su formación.

\section{Valoración de los expertos sobre las dimensiones que caracterizan la cultura matemática para su formación}

Con el objetivo de hacer una valoración de los aspectos que caracterizan la cultura matemática, se sometió a la consideración del criterio de expertos un conjunto de dimensiones, que se corresponden con la generalidad de las expresadas en la literatura científica. Estas son: la dimensión cognitiva, la dimensión histórica, la dimensión de aplicabilidad, la dimensión perimatemática y la dimensión formativa.

Uno de los métodos más utilizados que forman parte de las investigaciones en diferentes esferas, es el criterio de expertos, tiene aplicaciones importantes cuando se necesita recurrir a la opinión de expertos en el curso de una investigación. Autores como Crespo (2007) y Cruz y Campano (2008) hacen referencia a este método. Se aplicó el 'método de la comparación por pares'; con la utilización de las hojas de cálculo del tabulador electrónico Microsoft Office Excel, programadas con ese fin.

Se consideró la población de docentes de la Universidad de Cienfuegos, los Institutos Politécnicos y profesionales jubilados (docentes e investigadores) de la misma ciudad, se tuvo en cuenta los criterios de selección, años de experiencia como docentes en la enseñanza de la matemática, título académico, disposición de colaborar en el trabajo dado su creatividad, buena capacidad de análisis, espíritu crítico y autocrítico, y un desempeño profesional destacado.

Para la selección de los expertos se aplicó a una muestra intencional de 36 docentes, con el objetivo de determinar el nivel de competencia de los expertos.

El coeficiente de competencia se calculó por la siguiente fórmula: $K=1 / 2(k c+k a)$, donde $(k c)$ es el coeficiente de conocimiento o información que tiene el experto acerca de la formación de la cultura matemática y ( $\mathrm{ka}$ ) es el coeficiente de argumentación o fundamentación de los criterios del experto sobre el tema. Para determinar la competencia se tuvo en cuenta la escala valorativa siguiente: si o, $8<\mathrm{K}<1,0$ el coeficiente de competencia es alto; si $0,5<\mathrm{K}<0,8$ el coeficiente de competencia es medio; y si $\mathrm{K}<0,5$ el coeficiente de competencia es bajo.

El procesamiento de los resultados llevó a la conclusión de que 22 docentes tienen un coeficiente de competencia alto, 8 de medio y 6 tienen un coeficiente de competencia bajo. En 
resumen, 30 docentes fueron declarados como expertos al considerar la posibilidad de tomar también a los 8 docentes con el coeficiente de competencia medio. Todos son universitarios, el $86,6 \%$ son licenciados y el resto ingenieros, 25 son Máster o Doctores y el $30 \%$ pertenece a esta última categoría, el resto no tiene ningún título académico.

Después de la determinación de la cantidad de expertos, confección del listado; y haber obtenido el consentimiento del experto en su participación, se comenzó con la primera ronda. Con el objetivo de valorar las dimensiones que caracterizan la cultura matemática para su formación en los estudiantes de la Licenciatura en Educación, Matemática, se aplicó el cuestionario con las dimensiones cognitiva, histórica, de aplicabilidad, perimatemática y formativa, y los rasgos que la caracterizaban.

Para valorar las dimensiones se utilizaron cinco categorías evaluativas: (1) sin criterio, (2) eliminar, (3) sustituir, (4) modificar y (5) no modificar, la aplicación del instrumento a los expertos se efectúo en dos rondas de análisis crítico y rediseño de las dimensiones hasta lograr que obtuviera niveles de aceptación. Los resultados de la valoración por los expertos en la primera ronda se procesaron estadísticamente.

Los expertos señalaron No modificar y Eliminar en las dimensiones propuestas, opinaron y sugirieron modificaciones para mejorarlas, sin señalar dimensiones adicionales. La mayoría de los valores, resultaron menores que el punto de corte de esa categoría, o sea, $\mathrm{N}-\mathrm{P}<2,5589$.

Tabla 1

Categorías y rango de valoración de las dimensiones de la cultura matemática

\begin{tabular}{|l|c|c|}
\hline \multicolumn{3}{|c|}{ Puntos de corte } \\
\hline \multicolumn{1}{|c|}{ Categorías evaluativas } & Mayor que & $\begin{array}{c}\text { Menor o igual } \\
\text { que }\end{array}$ \\
\hline No modificar & & 2,5589 \\
\hline Modificar & 2,5589 & 3,1898 \\
\hline Sustituir & 3,1898 & 3,3258 \\
\hline Eliminar & 3,3258 & 4,2649 \\
\hline Sin criterio & 4,2649 & \\
\hline
\end{tabular}

Tabla 2

Resultado de la valoración de las dimensiones de la cultura matemática

\begin{tabular}{|l|c|c|}
\hline \multicolumn{3}{|c|}{ Dimensiones de la cultura matemática } \\
\hline Cognitiva & N-P & Categoría \\
\hline Histórica & $-0,9301$ & No modificar \\
\hline De aplicabilidad & $-0,9301$ & No modificar \\
\hline Perimatemática & $-0,9301$ & No modificar \\
\hline Formativa & 3,7202 & Eliminar \\
\hline
\end{tabular}

Además, se determinó el grado de acuerdo entre los expertos, con la prueba no paramétrica del coeficiente de concordancia $\boldsymbol{\omega}$-Kendall, con $\mathrm{N}$ muestras relacionadas, según los requisitos de la prueba, mediante el diseño estadístico. El cálculo se realizó con el paquete esta- 
dístico SPSS para Windows, versión 20.0. El coeficiente $\omega$-Kendall funciona como medida de relación entre $\mathrm{k}$ ordenaciones que pueden ser ligadas de $\mathrm{m}$ objetos o individuos, en $\mathrm{N}$ muestras relacionadas. El coeficiente de concordancia $\omega$-Kendall resultó de 75,7\%, o sea alto en la escala asumida y significativo, ya que , lo cual indica que puede rechazarse la hipótesis nula.

Tabla 3

Dimensiones para analizar la concordancia de los expertos

\begin{tabular}{|l|c|}
\hline \multicolumn{2}{|c|}{ Rangos } \\
\hline & $\begin{array}{c}\text { Rango } \\
\text { promedio }\end{array}$ \\
\hline Cognitiva & 3,70 \\
Histórica & 3,50 \\
De aplicabilidad & 3,30 \\
Formativa & 3,48 \\
Perimatemática & 1,02 \\
\hline
\end{tabular}

Tabla 4

CONCORDANCIA ENTRE LOS EXPERTOS

\begin{tabular}{l} 
Estadísticos de contraste \\
\begin{tabular}{|l|c|}
\hline $\mathrm{N}$ & 30 \\
W de Kendalla & 757 \\
Chi-cuadrado & 90,859 \\
gl & 4 \\
Sig. asintót. &, 000 \\
\hline
\end{tabular} \\
a. Coeficiente de concordancia \\
\multicolumn{2}{|c|}{ de Kendall }
\end{tabular}

Las descripciones correspondientes a cada dimensión se modificaron de acuerdo con los cambios sugeridos siguientes:

- Incorporar en la descripción de la dimensión de aplicabilidad los elementos sobre arte, medicina, medio ambiente, construcciones y otros.

- Eliminar la dimensión Peri-matemática que contiene lo relacionado con la matemática y el arte, los juegos, la naturaleza, que puede estar implícita en la dimensión de aplicabilidad.

- Incluir elementos de epistemología de las matemáticas y las vertientes filosóficas de la matemática (Platonismo, logicismo, formalismo y constructivismo) en una de las dimensiones.

- De manera general reconocen la importancia de la formación de la cultura matemática, la necesidad de aspectos o dimensiones que pueden caracterizar la cultura matemática de un estudiante.

Los expertos en la segunda ronda valoraron las dimensiones cognitiva, histórica, de aplicabilidad y formativa, mediante cinco categorías ordinales. Se describieron los resultados me- 
diante el análisis de frecuencias absolutas, acumuladas, relativas acumuladas, y sus imágenes en la tabla de la inversa de la función de distribución normal. Además, se presentan los puntos de corte de las categorías a comparar con cada uno de los valores promedios de las variables valoradas. La mayor cantidad de encuestados señalaron la valoración Máxima, y del análisis de los puntos de corte se infirió que las valoraciones se ubicaran en la clasificación de No modificar. Todos los valores, resultaron menores que el punto de corte de esa categoría, o sea, N-P<3,6571.

Tabla 5

Categorías y rango de valoración de las dimensiones de la cultura matemática

\begin{tabular}{|l|c|c|}
\hline \multicolumn{3}{|c|}{ Puntos de corte } \\
\hline \multicolumn{1}{|c|}{ Categorías evaluativas } & Mayor que & $\begin{array}{c}\text { Menor o igual } \\
\text { que }\end{array}$ \\
\hline No modificar & & 3,6571 \\
\hline Modificar & 3,6571 & 4,2649 \\
\hline Sustituir & 4,2649 & 4,2649 \\
\hline Eliminar & 4,2649 & 4,2649 \\
\hline
\end{tabular}

Tabla 6

Resultado de la valoración de las dimensiones de la cultura matemática

\begin{tabular}{|l|c|c|}
\hline \multicolumn{3}{|c|}{ Dimensiones de la cultura matemática } \\
\hline & N-P & Categoría \\
\hline Cognitiva & $-0,1519$ & No modificar \\
\hline Histórica & $-0,1519$ & No modificar \\
\hline De aplicabilidad & 0,4558 & No modificar \\
\hline Formativa & $-0,1519$ & No modificar \\
\hline
\end{tabular}

En sentido general, las opiniones, recomendaciones y sugerencias fueron atendidas por la autora de esta investigación a fin de perfeccionar las dimensiones propuestas para la formación de la cultura matemática de los estudiantes de la Licenciatura en Educación, Matemática. Los expertos estuvieron totalmente de acuerdo con los cambios realizados.

Por lo que se considera como dimensiones que caracterizan la cultura matemática:

- La dimensión cognitiva: se expresa en los contenidos propios de las disciplinas específicas de la carrera, en las alternativas de solución a los problemas profesionales sustentados en la apropiación de los conocimientos, habilidades, valores, la logicidad del pensamiento, el enfoque interdisciplinario.

- La dimensión histórica: se expresa en conocimiento sobre la génesis y evolución de los descubrimientos y desarrollo de la ciencia matemática y su enseñanza.

- La dimensión de aplicabilidad: se expresa en la actividad práctica que realiza el sujeto con el fin de describir, predecir, prescribir, demostrar y transformar la realidad en diferentes contextos, utilizando la matemática. 
- La dimensión formativa: se expresa en los métodos, procedimientos, algoritmos, modos de actuación y valores.

Con la idea de mostrar un resultado, se ha valorado y reflexionado en cómo demostrar que es posible realizar el estudio de este fenómeno, a pesar de la amplitud, complejidad y diversidad de información contenida en él.

Teniendo en cuenta el proceso de enseñanza aprendizaje de las disciplinas específicas, las exigencias de los programas de las mismas y el modelo del profesional de la Licenciatura en Educación, Matemática para la formación de la cultura matemática, se identificarán las tareas docentes en correspondencia con los conocimientos de las disciplinas y las dimensiones a relacionar con los mismos, para formar desde el punto de vista personal y profesional la cultura matemática y general e integral del estudiante.

\section{Conclusiones}

El análisis teórico muestra que los aspectos esenciales que han de considerarse en la formación de la cultura matemática se manifiestan de forma fragmentadas por diferentes autores. Además, no se considera estos aspectos unificados en la formación del docente para su desempeño profesional y su cultura general integral.

La realización de este trabajo permitió precisar mediante la colaboración de los expertos, las dimensiones concretas y suficientes para formar la cultura matemática, al considerar la dimensión perimatemática contenida en la dimensión de aplicabilidad, según los rasgos que caracterizan a esta última.

Se considera que las dimensiones cognitiva, histórica, de aplicabilidad y formativa caracterizan la cultura matemática. Se puede contribuir a su formación mediante vías metodológicas o didácticas aplicadas en el proceso de enseñanza aprendizaje de la Licenciatura en Educación Matemática, para llevar a cabo la misión del profesor de contribuir con la formación cultural del estudiante. 
Álvarez, C. (1999). La Escuela en la vida. Didáctica. La Habana: Félix Varela.

Arteaga, E. (2017). La historia de la matemática en la educación matemática. Revista Conrado, 13(59), 62-68.

Bello, G., Machado, M. I., \& López, P. A. (2015). Estrategia didáctica para desarrollar la cultura económica en estudiantes de Preuniversitario. Boletín de la Sociedad cubana de Matemática y Computación. Congreso internacional COMPUMAT 2015. La Habana: Universidad de Ciencias Informáticas.

Bishop, A. (1999). Enculturación Matemática: La educación matemática desde una perspectiva cultural . (Sánchez, Trad.) Barcelona, México: Paidós.

Blanco, H., Fernández, A., \& Oliveras, M. L. (2017). Formación de profesores de matemática desde la Etnomatemática: estado de desarrollo. Revista Bolema, 31(58), 564-589.

Camarena, P. (enero-marzo de 2009). La matemática en el contexto de las ciencias. Revista Redalyc, 9(46), 15-25. México, Distrito Federal: Innovación Educativa.

Cantoral, R. (2013). Matemática educativa y las matemáticas del planeta Tierra 3. Barcelona: Cinvestav.

Carralero, L., Tamayo, M. A., \& Alonso, L. A. (2013). La cultura económica en el sistema educativo cubano. Educación y conciencia económica. 19(1). Ciencias Holguín.

Coriat, M., \& Flores, P. (mayo/agosto de 1998). La formación de profesores de matemática y la cultura escolar. Interuniversitaria de formación del profesorado(32), 25-37.

Crespo, T. (2007). Respuestas a 16 preguntas sobre el empleo de expertos en la investigación pedagógica. LIMA, Perú: Editorial San Marcos.

Cruz, M., \& Campano, A. (2008). El procesamiento de la información en las investigaciones educacionales. La Habana: Educación Cubana.

De Guzmán, M. (1993). Tendencias innovadoras en educación matemática. Cátedra Miguel de Guzmán. Madrid: Facultad CC Matemáticas - UCM.

Fernández, B. (2017). Alternativa didáctica para la motivación del estudio de la carrera Licenciatura en Educación. Especialidad Matemática - Física. [tutores: A. García y E. Arteaga] . Tesis en opción al título de Máster en Educación. Cienfuegos: Universidad "Carlos Rafael Rodríguez" de Cienfuegos.

García, O., Buitrago, H., \& Cañon, C. (2015). Aplicaciones tecnológicas en la enseñanza del Cálculo. Congreso Internacional COMPUMAT 2015.

Horruitiner, P. (2008). La Universidad Cubana: el modelo de formación. La Habana: Félix Varela.

León, M. (1999). La historia de la Matemática como recurso motivacional en la enseñanza de la Matemática. Tesis en opción al título de Máster en Educación [Tutor: M.M. López]. Cienfuegos.

Lezama, J., \& Mariscal, E. (1995). Docencias en Matemáticas: hacia un modelo del profesor desde la perspectiva de la socioepistemología. Comité Latinoamericano de Matemática Educativa A. C.

Mingüer, L., \& Lezama, J. (2007). Entorno sociocultural y cultura matemática en profesores del nivel superior de educación. Estudio de caso en el Instituto Tecnológico de Oaxaca. Una aproximación socioepistemológica. Resultados. Acta Latinoamericana de Matemática Educativa, 20, 560- 566.

Molina, C. M. (agosto de 2003). Reseña de "Conceptuación del término cultura matemática en el nivel secundaria". Revista Educación Matemática, 15(002), 185-188.

Moreno, M. (2017). El desarrollo de la cultura geográfica en estudiantes de secundaria básica. Tesis en opción al grado científio de Doctor en Ciencias Pedagógicas. Santa Clara: Universidad central "Marta Abreu" de las Villas.

Núñez, J. (1999). La ciencia y la tecnología como procesos sociales: Lo que la educación científica no debería olvidar. La Habana: Félix Varela. 
Palanqué, R. L., Toledo, T. M., Torres, I. C., Cables, O., \& Almaguer, E. (2015). La Matemática y su enseñanza para la cultura económica en la formación del profesional de la especialidad Matemática - Física. Boletín de la Sociedad cubana de Matemática y Computación. Congreso internacional COMPUMAT 2015. La Habana: Universidad de Ciencias Informáticas.

PISA/OECD. (2004). La evaluación de la "Cultura Matemática" en PISA 2003. Marco conceptueal y actividades de las pruebas. Montevideo: Gerencia de Investigación y Evaluación.

Pupo, N. (2006). El desarrollo de la cultura energética en estudiantes de secundaria básica, mediante una concepción didáctica integradora.[tutor: A. Roca; F. Estrada]. Tesis en opción al grado científico de Doctor en Ciencias Pedagógicas. Holguín.

Raya, G. (2002). La cultura política como factor que incide en el voto en los procesos electorales de México. [tutor: O. E.A. Cuell]. Tesis para obtener el título de Licenciado en Sociología. México.

Rocío, E. G. (7 de noviembre de 2016). La técnica de enseñanza de matemáticas en China es la mejor, según estudio. Otras voces en Educación(3).

Rodríguez, V., Correa, A., \& Hernández, D. (2014). Implementación de una herramienta para el cálculo de un enlace por fibra óptica. Boletín de la Sociedad cubana de Matemática y Computación. Congreso internacional COMPUMAT 2015.

Salazar, D. (2004). Cultura científica y formación interdisciplinaria de los profesores en la actividad científico-investigativa. En F. Addine, Didáctica: teoría y práctica. La Habana: Pueblo y Educación.

Soto, J. (2015). La Didáctica de la Matemática vista desde la Facultad de Ciencias de la Universidad de Chile. Revista Anales, Séptima Serie(8).

Tones, J. (2015). 14 obras de Escher que nunca nos cansamos de ver. Revista El País.

Valdivia, I. (2012). La formación de la cultura política desde el Marxismo Leninismo.[Tutores: S.Arteaga y M. Cárdenas]. Tesis en opción al grado científico de Doctor en Ciencias Pedagógicas. Villa Clara: Universidad de Ciencias Pedagógicas "Félix Varela" de Villa Clara. 